\title{
Cultured human astrocytes secrete large cholesteryl ester- and triglyceride- rich lipoproteins along with endothelial lipase
}

Lin Yang*, Yanzhu Liu*§, Trudy M. Forte*, Jeffrey W. Chisholm», John S. Parks", and Neil S. Shachter*

Abbreviated Title: Human astrocytes secrete VLDL-sized lipoproteins

*Department of Medicine, Columbia University, New York, NY

${ }^{*}$ Life Sciences Division, Lawrence Berkeley National Laboratory, Berkeley, CA

TDepartment of Pathology, Wake Forest University School of Medicine, Winston-Salem, NC

${ }^{\S}$ Yanzhu Liu and Lin Yang contributed equally.

Correspondence should be directed to Dr. Neil S. Shachter, Department of Medicine, Columbia University, 630 W. 168th Street, PH 10-305, New York, NY 10032. Telephone: (212) 305-9893, Facsimile: (212) 305-3213, Electronic mail: nss5@columbia.edu 


\begin{abstract}
We cultured normal human astrocytes and characterized their secreted lipoproteins. Human astrocytes secreted lipoproteins in the size range of plasma VLDL (Peak 1), LDL (Peak 2), HDL (Peak 3) and a smaller peak (Peak 4), as determined by gel filtration chromatography, nondenaturing gradient gel electrophoresis and transmission electron microscopy. Cholesterol enrichment of astrocytes led to a particular increase in Peak 1. Almost all Peak 2, 3 and 4 cholesterol and most Peak 1 cholesterol was esterified (unlike mouse astrocyte lipoproteins, which exhibited similar peaks but where cholesterol was predominantly non-esterified). Triglycerides were present at about $2 / 3$ the level of cholesterol. LCAT was detected along with two of its activators, apolipoprotein (apo) A-IV and apoC-I. ApoA-I and apoA-II mRNA and protein were absent. ApoJ was present equally in all peaks but apoE was present predominantly in peaks 3 and 4. ApoB was not detected. The electron microscopic appearance of Peak 1 lipoproteins suggested partial lipolysis leading to the detection of a heparin-releasable triglyceride lipase consistent with endothelial lipase. The increased neuronal delivery of lipids from large lipoprotein particles, for which apoE4 has greater affinity than does apoE3, may be a mechanism whereby the apoE $\varepsilon 4$ allele contributes to neurodegenerative risk.
\end{abstract}

Key words: Lipoproteins, VLDL; Apolipoproteins C; Apolipoproteins E; Cell Culture; Astrocytes; Human

Abbreviations used in this paper: Alzheimer's Disease, AD; apo, apolipoprotein; central nervous system, CNS; cerebrospinal fluid, CSF; Electron Microscopy, EM; glial fibrillary acidic protein, GFAP; lecithin:cholesterol acyltransferase, LCAT; normal human astrocytes, NHA; triglyceride, TG. 


\section{INTRODUCTION}

Central nervous system (CNS) lipoproteins are primarily secreted by astrocytes, but are also secreted by microglia $(1,2)$. CNS lipoproteins obtained from the cerebrospinal fluid (CSF) have been found by most observers to be small $(7-15 \mathrm{~nm})$ and in the HDL density range, $d>1.063-1.21 \mathrm{~g} / \mathrm{ml}$ (3-6). However, there has been a report of larger, LDL-density (mean diameter $18.3 \mathrm{~nm}, d=1.006-1.06 \mathrm{~g} / \mathrm{ml}$ ) apoE-containing lipoproteins in human CSF (7). Lipoproteins from human CSF are cholesteryl ester-enriched and induce cholesterol efflux (3-5). The latter is likely accounted for by their apoE component (8). In contrast, cultured rodent astrocytes, including those from human apoE3 and human apoE4 transgenic mice, have been reported to secrete HDL discs that are rich in free cholesterol $(1,3)$. Cholesteryl esters in the human lipoproteins may be generated after secretion due to the actions of the lecithin:cholesterol acyltransferase (LCAT) that is known to be present in brain (6). However, the characteristics of the lipoproteins secreted by human astrocytes in culture have not been described and may account for the observed differences between human CSF lipoproteins and those secreted by cultured rodent astrocytes.

The apolipoprotein (apo) E genotype, principally the $\varepsilon 2 / \varepsilon 3 / \varepsilon 4$ allelic isoforms, is the most important genetic predictor of late-onset Alzheimer's Disease (AD) but the mechanism of this observation remains uncertain $(9,10)$. ApoE is a constituent of central nervous system (CNS) lipoproteins that binds lipoprotein receptors and plays an important role in the distribution of CNS lipid $(11,12)$. The composition of the lipoproteins secreted by human astrocytes may shed light on the mechanism by which the apoE allelic variants alter risks of $\mathrm{AD}$ and of post-traumatic brain injury (10). CSF lipoproteins, more properly considered "lipoprotein remnants," likely do 
not reflect the lipoproteins to which neurons are exposed in the brain interstitium. We, therefore, examined the size profile, electron microscopic appearance and the lipid and apolipoprotein composition of lipoproteins secreted by normal human astrocytes in culture. 


\section{MATERIALS AND METHODS}

\section{Cell Culture}

Normal human astrocytes (NHA) from a single donor were purchased from Clonetics (Catalogue \#CC-2565, BioWhittaker, Inc., 8830 Biggs Ford Road, Walkersville, MD 21793-0127). This supplier warranted that "the NHA test positive for GFAP, negative for CD68 and negative for CNPase. In order to pass quality control the cells must be $=90 \%$ for GFAP, an astrocyte marker." The cells (passages 3-6) were grown in Astrocyte Growth medium (AGM CC-3186) which included Astrocyte Basal Medium (ABM CC-3187) and supplements of $20 \mathrm{ng} / \mathrm{ml} \mathrm{hEGF}$ (CC-4107), $25 \mu \mathrm{g} / \mathrm{ml}$ insulin (CC-4108), $25 \mathrm{ng} / \mathrm{ml}$ progesterone (CC-4106), $50 \mu \mathrm{g} / \mathrm{ml}$ transferrin (CC-4109), $50 \mu \mathrm{g} / \mathrm{ml}$ gentamicin, $50 \mathrm{ng} / \mathrm{ml}$ amphotericin-B (CCC-4081), and 5\% $\mathrm{FBS}$ at $37^{\circ} \mathrm{C}$ in $5 \% \mathrm{CO}_{2}$. The cells were either treated or untreated with $16 \mu \mathrm{g} / \mathrm{ml}$ of cyclodextrin-solubilized cholesterol (Sigma Cat \# C-3045). This concentration of supplemental cholesterol was the highest that produced no change in astrocyte growth and viability.

Single-donor human astrocytes from two donors were also purchased from Clonexpress (Catalogue \# HAST O40, Clonexpress, Inc., 504 E. Diamond Ave, Suite G, Gaithersburg, MD 20877). The cells (passages 3-8) were grown in Astrocyte Growth Medium that included Basal Medium (D-MEM/F12, Gibco-BRL Catalogue \# 12400-024), 10\% fetal bovine serum and Astrocyte Cell Supplement (Clonexpress). Greater than 80\% glial fibrillary acidic protein (GFAP) staining positivity was documented by the supplier.

We independently tested all cells for GFAP immunofluorescence, as described (13), using 1:100 Rat-GFAP (Calbiochem, \#345860), and documented greater than 90\% GFAP positivity in all cases (data not shown). 


\section{Mouse astrocyte culture}

Mouse astrocyte cultures were established from individual neonatal C57BL/6J mice, as described (14). Cells from the third or fourth passage were cultured in IMDM without FBS for 48 hours. Media were collected and concentrated for FPLC, as above.

\section{ApoE genotyping}

DNA was extracted from the astrocytes and frozen at $-20^{\circ} \mathrm{C}$. Genotype for the $\varepsilon 2, \varepsilon 3$ and $\varepsilon 4$ alleles of apoE was determined as described (15).

\section{RNase Protection Assay}

Total RNA from human astrocytes was prepared by the acid guanidinium thiocyanate method (16). The human apoC-I cRNA probe was prepared as described previously (17). Forty $\mu \mathrm{g}$ of human astrocyte RNA (4th passage) and $30 \mu \mathrm{g}$ of RNA from the brain of a human apoC-I transgenic mouse were hybridized at $65^{\circ} \mathrm{C}$ to the human apoC-I ${ }^{32} \mathrm{P}$-labeled antisense RNA probe using a kit (catalogue \#1414, Ambion) overnight. A ${ }^{32} \mathrm{P}$ labeled antisense 18S rRNA probe was also used as a control for RNA amount. Residual unhybridized RNA was degraded using the kit reagents. Reaction products were separated by 5\% PAGE. The protected fragment was quantitated by exposure to a PhosphorImager and ImageQuaNT software (Molecular Dynamics, Sunnyvale, CA).

\section{Lipoprotein isolation}

When astrocytes were confluent, media were removed and the cells were washed twice with HEPES Buffered Saline Solution (Bio Whittaker, Inc. catalogue \#CC-5022). All cultures were 
then incubated in either $10 \mathrm{ml}$ of serum free AGM or DMEM/F12 (second and third donor) without supplemental cholesterol for 72 hours. Conditioned media were collected and clarified by centrifugation at $5000 \times \mathrm{g}$ for 10 minutes. For lipid analyses, the medium from ten T75 flasks was collected and then concentrated to $0.5 \mathrm{ml}$ with Centriplus or Centricon YM10 (Millipore, Bedford, MA Cat.\# 4411 or 4241) (). The entire $0.5 \mathrm{ml}$ was then fractionated in $0.15 \mathrm{M} \mathrm{NaCl}, 1$ mM EDTA, pH 8.0 by gel filtration chromatography (FPLC, Amersham Pharmacia Biotech. 2636 South Clearbrook Dr. Arlington Heights, IL 60005) using two Superose-6 columns in series (Cat \# 17-0537-01). Fifty fractions of $500 \mu \mathrm{l}$ each were collected and analyzed.

\section{Lipid assays}

Total cholesterol (Sigma PO box 14508 St. Louis, MO Cat \# 352-20), Free Cholesterol (Cat \# 274-47019, Wako, 1600 Bellwood Road Richmond, VA) and Triglycerides (Sigma Cat \# 33920) were measured using kits.

\section{Apolipoprotein Immunoblot Analysis}

SDS-PAGE was performed under reducing conditions. The concentrated media (see above) were fractionated either by $10 \%$ SDS-PAGE or $16 \%$ SDS-PAGE in Tris-Tricine buffer. Proteins were electrophoretically transferred to nitrocellulose membrane and probed with 1:5000 antihuman apoC-I (Cat. \# K74110R, BioDesign, 60 Industrial Park Road, Saco, ME), 1:5000 antihuman apoA-I (Cat \# K45252G, BioDesign), 1:8000 anti-human apoA-II (Cat \# K34001G, BioDesign), 1:12,000 anti-human apoA-IV (gift of Charles L. Bisgaier) (18), 1:5000 anti-human apoJ (Cat \# K59198G, BioDesign), 1:5000 anti-human apoE (Cat \# A40720, Transduction 
Laboratories, 133 Venture Court Lexington, KY 40511-2624) or 1:1000 anti Apo E (BD Transduction Laboratories).

For the apoA-I, apoA-II immunoblots, the serum free media were concentrated 100-fold using Centricon YM-3 filtration devices (Millipore, Bedford, MA Cat. \#4202). Ten 15- $\mu 1$ aliquots were loaded; 0.2-1 $\mu 1$ aliquots of human plasma were used as positive controls. For the apoE and apoJ immunoblots, constant amounts of total protein were loaded. For the apoA-IV immunoblot, human astrocytes were treated or untreated with $16 \mu \mathrm{g} / \mathrm{ml}$ of cholesterol as above. Serum-free conditioned media (48 hours) were concentrated about 100 fold. One hundred $\mu \mathrm{g}$ of protein was used for both control and cholesterol-treated media. For the apoC-I immunoblots of FPLC fractions, $100 \mu 1$ from each of the $1^{\text {st }}-13^{\text {th }}$ FPLC fractions was pooled, as were $100-\mu 1$ aliquots from the $31 \mathrm{st}-49^{\text {th }}$ fractions. The two pools were separately concentrated to $10 \mu 1$ using a Microcon YM-3 (Millipore, Cat \# 42420) and the entire $10 \mu$ was fractionated by SDS-PAGE and transferred to a nitrocellulose membrane.

\section{Apolipoprotein B Immunoblot}

FPLC was performed on $500 \mu 1$ of Centricon-concentrated medium from 10 flasks of confluent human astrocytes. One hundred $\mu 1$ from each of fractions 1-13 and 31-50 fractions were pooled (separately). The two pools were each concentrated to about $10 \mu 1$ and were electrophoresed by

4\% SDS-PAGE along with positive (human LDL) and negative (medium) controls. Anti-human apoB1:3000 (Boehringer Mannhem,Catalogue \#7 26494) was applied to an immunoblot of the gel according to the manufacturer's instructions. 


\section{LCAT Immunoblot}

The LCAT polyclonal antibody was produced in 3 rabbits immunized with a total of $12 \mathrm{mg}$ of recombinant hLCATH6, C-terminal His6 tagged LCAT produced in stably transfected CHO cells (19). Screening of the antisera by western blotting indicated that the antisera from all three rabbits was similarly reactive towards both purified plasma LCAT and hLCATH6. The antibody was further affinity purified from a 3 rabbit anti-sera pool using an Affi-gel 15 column containing approx 1-2 $\mathrm{mg} / \mathrm{ml}$ of immobilized hLCATH6. The antibody was eluted from the column with glycine $\mathrm{HCl} \mathrm{pH} 2.8$, buffer-exchanged into PBS and stored at $-80^{\circ} \mathrm{C}$ in $2 \mathrm{mg} / \mathrm{ml}$ aliquots.

For the human LCAT immunoblot, serum-free medium conditioned by human astrocytes for $48 \mathrm{~h}$ was concentrated 100 fold and $4 \mu \mathrm{l}$ was fractionated by SDS-PAGE; one $\mu 1$ of human plasma was used as a positive control. After transfer, membranes were incubated with 1:700 affinity purified rabbit anti-human LCAT.

For the mouse LCAT immunoblot, serum-free medium conditioned by mouse astrocytes for $48 \mathrm{~h}$ was concentrated 100 fold and 4-8 $\mu 1$ was loaded on the gel. One $\mu 1$ of mouse plasma was used as a positive control.

\section{Lipoprotein Gradient gel Electrophoresis and Transmission Electron Microscopy (EM) Lipoprotein sizing by non-denaturing polyacrylamide gradient gel electrophoresis (20) and visualization and sizing by transmission EM (21) were performed as described.}




\section{Lipase Activity Assays}

Confluent cells were washed three times with PBS and then cultured overnight either in serumfree medium or serum-free medium supplemented with $100 \mathrm{units} / \mathrm{ml}$ heparin. The medium was collected and concentrated 100-fold via Centricon filtration. Triglyceride (TG) lipase activity assays were performed as described (22).

\section{Reverse Transcriptase (RT)-PCR}

Two $\mu \mathrm{g}$ total RNA of human astrocytes and $0.2 \mu \mathrm{g}$ each of control RNAs consisting of human liver RNA (Ambion, catalog \# 7960), human placental RNA (Ambion, catalog \# 7950) and total RNA extracted from heart tissue of a heart-expressing human lipoprotein lipase transgenic mouse (23) were applied for reverse transcription at $42^{\circ} \mathrm{C}$ for 30 minutes (Stratagene, Catalogue \# 600166). PCR was performed with specific primers based on the sequences of three different secreted heparin-releasable TG lipases. For lipoprotein lipase (Gen-Bank M15856), 5'GACACTTGCCACCTCATTCCC-3 and 5'-CATCAGGAGAAAGACGACTCGG-3' were used, amplifying a 450-bp cDNA. For hepatic lipase (Gen-Bank J03540), 5'GGTGGAACGCACAAGATTGG-3' and 5'-CTGGGTGATGGCATTGAAGC-3' were used, amplifying a 276-bp cDNA. For endothelial lipase (Gen-Bank AF118767), 5'-GGAGCCTCTGCTATTGCTTTGC-3' and 5'-GCCAGTCAACCACAACTACATTGG-3' were used, amplifying a 339-bp cDNA. The amplification profile was 5 minutes at $95^{\circ} \mathrm{C}$ for an initial denaturation, followed by 30 cycles of $95^{\circ} \mathrm{C}$ for $30 \mathrm{sec}, 56^{\circ}$ (lipoprotein lipase and endothelial lipase) or $60^{\circ} \mathrm{C}$ (hepatic lipase) for $30 \mathrm{sec}, 72^{\circ} \mathrm{C}$ for $1 \mathrm{~min}$. and final extension at $72^{\circ} \mathrm{C}$ for $10 \mathrm{~min}$. 


\section{RESULTS}

\section{Human Astrocyte Lipoproteins-Lipids}

Normal human astrocytes from Donor 1 (apoE genotype 3/4) were cultured in control medium or in medium supplemented with $16 \mu \mathrm{g} / \mathrm{ml}$ solubilized cholesterol. The apoE genotype of the astrocytes was $\varepsilon 3 / \varepsilon 4$. Lipoprotein profile was determined by gel filtration chromatography of serum-free conditioned medium. Under control conditions, cholesterol was evident in large particles (Peak 1) that eluted at the same fractions as does human plasma VLDL in this system, in smaller particles (Peak 2) that eluted at about the same fractions as does plasma LDL, in a third peak (Peak 3) that eluted at the same fractions as the major peak of plasma HDL and a fourth, smaller lipoprotein peak (Peak 4). About 2/3 of the cholesterol in Peak 1 was esterified and the cholesterol in the other fractions was almost completely esterified (Fig. 1a). When supplemental cholesterol was added to the medium there was a marked increase in cholesterol in lipoproteins, particularly in Peak 1 (Fig. 1b).

Triglyceride (TG) levels in lipoproteins in medium fractions from untreated cells were about $2 / 3$ the level of total cholesterol but increased only slightly with cholesterol treatment (Fig. 1c).

To confirm these findings, gel filtration chromatography was repeated on medium conditioned by astrocytes obtained from other donors (Donor 2 of $A P O E$ genotype $\varepsilon 3 / \varepsilon 4$ and Donor 3 of $A P O E$ genotype $\varepsilon 2 / \varepsilon 3)$. Though there were similarities between Donor 1 and Donors 2 and 3, such as the prevalence of CE-rich VLDL, each pattern showed distinct characteristics. In Donor 2 (Fig. 2a) Peak 1, both for cholesterol and TG, was more prominent than Peaks 3 and 4. Donor 2 exhibited a more prominent Peak 3 than Peak 4, while Donors 1 and 3 exhibited a relative preponderance of Peak 4 lipoproteins. In Donor 2, TG was principally detected in 
Peak 1, where it was present at about $1 / 3$ the level of cholesterol. A small amount of TG (equal to cholesterol) was present in Peak 4. Gel filtration chromatography of Donor 3 astrocyte medium (Figure $2 b$ ) revealed four peaks that were about equal in size, corresponding to the same four locations. TG was present at about half the level of cholesterol in Peak 1, present equally to cholesterol in Peak 2 and in Peak 3 and present at about double the level of cholesterol in Peak 4.

\section{Human Astrocyte Lipoproteins-Apolipoprotein Composition}

We were struck by the large amounts of cholesteryl ester in the secreted Peak 1, and its predominant presence in Peak 3, raising the question of whether extracellular cholesterol esterification in Peak 3 could be occurring, as it does in plasma lipoproteins of this size (HDL). An immunoblot of serum-free conditioned medium from Donor 1 revealed the presence of human lecithin:cholesterol acyltransferase (LCAT), the enzyme responsible for cholesterol esterification in plasma (Fig. 3a). We then examined the medium for activators of LCAT. ApoA-I, the major plasma activator of LCAT, and apoA-II were undetectable (data not shown) but a small amount of apoA-IV was present (Fig. 3b).

We also examined Donor 1 medium for apoC-I, another activator of LCAT. ApoC-I was clearly detected by immunoblot in concentrated astrocyte conditioned medium (data not shown). We then assayed for the presence of apoC-I in the concentrated FPLC fractions (Fig. 3c). The apoC-I signal in Peak 3 and 4 from medium from cholesterol-treated cells was $0.6 \mathrm{X}$ that in Peaks 3 and 4 from control medium. The apoC-I signal in Peak 1 isolated from medium conditioned by cells that had been cholesterol-treated was $0.45 \mathrm{X}$ that in control Peak 1. RNase protection assay confirmed the presence of apoC-I mRNA in the Donor 1 astrocytes (Fig. 4). Interestingly, 
apoC-I mRNA levels were much higher in the cholesterol-treated cells, indicating a probable post-transcriptional mechanism for the decrease in apoC-I protein that we observed.

ApoJ was also present in Donor 1 astrocyte-conditioned medium and increased 2.2-fold with cholesterol enrichment (Fig. 5a). ApoE (normalized for protein) increased 7.4-fold with cholesterol enrichment (Fig. 5b). ApoE was also assayed in both Peak 1 and Peak 3/4 FPLC peaks isolated from the second astrocyte donor (Fig. 6a). ApoE was disproportionately present in HDL, with only a small amount detectable in Peak 1. ApoE, ApoJ and apoA-IV were assayed in FPLC peaks isolated from Donor 3 (Fig. 6b). All three apolipoproteins could be detected in all four peaks except for Peak 2, where no apoE could be detected. No apoB was detectable, neither in concentrated astrocyte-conditioned media nor in concentrated FPLC fractions (data not shown).

\section{Human Astrocyte Lipoproteins Particle Sizing by Gradient Gel Electrophoresis and Transmission Electron Microscopy (EM)}

The Peak 1 peak as well as the pooled Peaks 3 and 4 from the second donor were examined by non-denaturing gradient gel electrophoresis and EM. The Peak 1 lipoproteins were heterogeneous in size and morphology by EM (Fig. 7a). Interestingly, many of the larger particles were vesicular-shaped and some particles were dumbbell-shaped (arrows), consistent with the presence of partially lipolyzed particles. By transmission EM the Peak 1 particles ranged in size from $40 \mathrm{~nm}$ to $180 \mathrm{~nm}$. The accompanying non-denaturing gradient gel profile shows the distribution only of the smaller FPLC Peak 1 particles (gradient-gel size peaks 1 and 2 were 40.3 and $38.3 \mathrm{~nm}$, respectively); the larger ones remained at the top of the gel. On nondenaturing gradient gels, the pooled FPLC Peaks 3 and 4 (Fig. 7b) exhibited three distinct size 
peaks at 9.9, 9.3 and $8.5 \mathrm{~nm}$, where the major components were those in size peaks 2 and $3(9.3$ and $8.5 \mathrm{~nm}$, respectively). Consistent with this observation, the majority of the FPLC Peaks 3 and 4 particles by EM were $9.4 \mathrm{~nm}$ in size.

\section{Presence of Lipase in Astrocyte-Conditioned Medium}

Because the EM appearance of Peak 1 was consistent with the partial lipolysis of lipoproteins, we assayed for TG lipase activity in concentrated astrocyte-conditioned medium from Donor 2. TG lipase activity in three pools of astrocyte-conditioned medium was $\sim 6$-fold elevated over background $(P<0.01)$. Levels were $2.39 \pm 0.65 \mathrm{nmol} \mathrm{FFA} / \mathrm{ml} / \mathrm{h}$ in cells cultured in nonheparinized medium vs. $4.29 \pm 1.31 \mathrm{nmol} \mathrm{FFA} / \mathrm{ml} / \mathrm{h}$ in three pools cultured in the presence of heparin $(P<0.02$, heparinized vs. non-heparinized).

To further characterize this heparin-releasable TG lipase activity, we performed reverse transcriptase PCR (RT-PCR) on total RNA extracted from Donor 1 cultured astrocytes. While no signal was detected for lipoprotein lipase or hepatic lipase mRNA, a strong signal was detected for endothelial-cell lipase mRNA (Fig. 8). The identity of this PCR product was confirmed by direct DNA sequencing of the isolated PCR product using the PCR primers (data not shown). A similarly strong endothelial lipase signal was obtained from Donor 2 astrocytes (data not shown).

\section{Mouse Astrocyte Lipoproteins: Lipids and Apolipoproteins}

We compared our findings with those obtained in our hands with lipoproteins isolated from primary mouse astrocyte cultures. Multiple such cultures could be established and gave reproducible results (Figure 9). Similar to our results with human astrocytes, 4 distinct peaks 
could be observed that were situated over about the same FPLC fractions. Cholesterol and TG levels were similar in all peaks. Peak 1, Peak 2 and Peak 4 were about equal in height with Peak 3 of smaller size. ApoE, apoJ and apoA-IV were assayed in pooled lipoproteins from all four peaks and gave results not too dissimilar to those in humans, when adjusted for the fact that the apoA-IV antibody used was less sensitive. However, unlike human astrocytes, apoE could be strongly detected in mouse Peak 3. Pooled lipoproteins peaks were assayed for total cholesterol and free cholesterol. Unlike the corresponding human lipoproteins, Peak 1 cholesterol was $47 \%$ esterified, Peak 2 was 54\%, Peak 3 only 34\% and Peak 4 39\%. Nevertheless, mouse astrocyte conditioned medium did have immunodetectible LCAT (Figure 10). 


\section{DISCUSSION}

We have characterized the lipoproteins secreted by normal human astrocytes in culture. Lipoproteins of all size classes were detected, with particles that eluted at the location of VLDL on FPLC and that were of the size of VLDL lipoproteins on non-denaturing gradient gel electrophoresis and EM. Such particles have not been previously described in human

preparations. This peak (Peak 1) was at least equal in area to Peaks 3 and 4 . Peaks 3 and 4 had FPLC elution characteristics, size by non-denaturing gradient gel electrophoresis and EM and lipid composition consistent with HDL, as has been described in reports of human CSF lipoproteins $(1,3-5)$. A "Peak 2" that eluted in the size range of LDL was also noted, and is consistent with a prior report of LDL-sized particles in human CSF lipoproteins (7). Despite these congruencies these lipoproteins were quite different than the corresponding plasma lipoprotein classes, most prominently by the absence of apoB and apoA-I.

Unlike the well described rodent astrocyte lipoproteins, cholesteryl esters and TG were prominent constituents of these lipoproteins, with TG present at about 2/3 the level of cholesterol. Almost all of the Peak 3 and 4 cholesterol and most of the Peak 1 cholesterol was esterified, possibly related to the presence of LCAT in human astrocyte medium along with two of its protein activators, apoC-I and apoA-IV $(24,25)$. Of note, LCAT has been reported to colocalize with human CSF apoA-IV, but not CSF apoA-I (5). ApoE also has some activity as an LCAT activator and may be contributing to the formation of esterified cholesterol (26). While we cannot be certain that the cholesteryl ester present in these particles was formed extracellularly, it is clear that all elements necessary for the extracellular esterification of cholesterol are present in the lipoproteins secreted by human astrocytes. However, we also detected LCAT and apoA-IV in the mouse astrocyte lipoproteins, despite the presence of a much 
lower degree of cholesterol esterification. This could reflect differences in the ability of mouse apoA-IV to activate LCAT. However, mouse apoA-IV transgenic mice have been reported to exhibit increased cholesterol esterification (27). Interestingly, despite the presumed dependency of VLDL-sized lipoprotein secretion on apoB (28), no apoB could be detected in concentrated astrocyte-secreted medium or FPLC fractions, indicating their apparent assembly via a novel biochemical mechanism.

We have also used our approach to characterize the lipoproteins secreted by mouse astrocytes. Similar to a prior report, we find that the cholesterol in mouse astrocyte "HDL" is primarily unesterified (1). However, in contrast to this report, the "VLDL" (Peak 1) and, especially, "LDL" (Peak 2) are more prominent in our hands. Moreover, in important contrast to earlier work (3), we do detect significant TG in mouse (and human) astrocyte lipoproteins, present at the same concentration as cholesterol in the mouse lipoproteins. The delivery of TG may be an important function common to mouse and human astrocyte lipoproteins. In contrast, efficient cholesterol esterification, and subsequent efficient cholesterol uptake, may be specific to human astrocyte lipoproteins and may explain the previously noted differences in composition between astrocyte lipoproteins (previously reported from rodents only) and human CSF lipoproteins (3).

We have previously observed an inverse relationship between apoC-I protein and apoC-I mRNA levels in human brain specimens, the basis of which was uncertain (29). We have now observed similar findings in isolated human astrocytes variably exposed to supplemental cholesterol. Unlike apoE and apoJ, which increased in lipoproteins in media from cells exposed to supplemental cholesterol, apoC-I did not increase and appeared to decrease. This observation may reflect a post-transcriptional mechanism operative in astrocytes whereby increased cellular 
lipid stores may negatively affect the translation of apoC-I mRNA. The homeostatic rationale for such a mechanism may be apoC-I's role as an activator of LCAT and inhibitor of lipoprotein binding to receptors (30), actions that facilitate the acceptance by lipoproteins of cellular cholesterol and decrease the receptor-mediated delivery of lipoprotein cholesterol (and TG) to cells. A decrease in astrocyte secretion of apoC-I would facilitate the delivery of astrocyte cholesterol to neurons whenever astrocyte cholesterol stores are ample. Altered regulation of this pathway would have an influence on neuronal lipid flux and could provide a direct mechanism for the observed association between a regulatory apoC-I polymorphism and $\mathrm{AD}$ risk $(31,32)$.

A heparin-releasable TG lipase activity was detected that was found to likely represent endothelial lipase. The predominant detection of smaller lipoproteins with low TG content, HDL, in human CSF lipoproteins may be reflective of the action of this lipase in vivo. Endothelial lipase has prominent phospholipase activity (33). Thus, along with delivering fatty acid, the action of this lipase would be expected to specifically facilitate the particulate uptake of the secreted lipoproteins $(34,35)$. Contamination by endothelial cells would appear to be an unlikely explanation for the presence of endothelial lipase, given culture conditions that do not favor the persistence of such cells (36). However, astrocytes have much in common with brain microvascular endothelial cells and no biochemical marker has been defined that can distinguish them $(36,37)$. Therefore, the presence of the endothelial lipase cannot be considered surprising.

The principal ligand for receptor-mediated lipoprotein uptake, apoE, was also observed in astrocyte-conditioned medium, as would be expected (38), though to a lesser extent on large particles than on small particles. The increase in apoE in media from cholesterol-treated cells is consistent with the increase in lipoprotein cholesterol observed under these conditions. The 
apoE4 allelic isoform has been documented to have greater affinity for large lipoprotein particles $(39,40)$. We have now shown that such large particles are secreted by human astrocytes and that their secretion increased when the astrocytes were loaded with cholesterol, as would occur under circumstances of neurodegeneration related to disease or trauma. The increased delivery of lipid from such particles to neurons, along with a subsequent increase in beta-amyloid generation mediated via decreased $\alpha$-secretase activity (41), may be a mechanism whereby the apoE $\varepsilon 4$ allele contributes to increased $\mathrm{AD}$. 


\section{Acknowledgements}

We thank David Sulzer for advice and critical review of the manuscript. This research was supported by NHLBI/NIH R01 HL56232 (NSS), by an American Heart Association, New York City Affiliate Grant-in-Aid (NSS), by a grant from the Roth Foundation (NSS), HL18574 (MF), NIH R01 HL-54176 (JSP) and AHA grant 9804829 U (JWC). 


\section{REFERENCES}

1. Fagan, A. M., D. M. Holtzman, G. Munson, T. Mathur, D. Schneider, L. K. Chang, G. S.

Getz, C. A. Reardon, J. Lukens, J. A. Shah, and M. J. LaDu. 1999. Unique lipoproteins secreted by primary astrocytes from wild type, apoE (-/-), and human apoE transgenic mice. J. Biol. Chem. 274: 30001-30007.

2. Xu, Q., Y. Li, C. Cyras, D. A. Sanan, and B. Cordell. 2000. Isolation and characterization of apolipoproteins from murine microglia. Identification of a low density lipoprotein-like apolipoprotein J-rich but E-poor spherical particle. J. Biol. Chem. 275: 31770-31777.

3. LaDu, M. J., S. M Gilligan., J. R. Lukens, V.G. Cabana, C. A. Reardon, L. J. Van Eldik, and D. M. Holtzman. 1998. Nascent astrocyte particles differ from lipoproteins in CSF. $J$. Neurochem. 70: 2070-2081.

4. Rebeck, G. W., N. C. Alonzo, O. Berezovska, S. D. Harr, R. B. Knowles, J. H. Growdon, B. T. Hyman, and A. J. Mendez. 1998. Structure and functions of human cerebrospinal fluid lipoproteins from individuals of different APOE genotypes. Exp. Neurol. 149: 175-182.

5. Demeester, N., G. Castro, C. Desrumaux, C. De Geitere, J. C. Fruchart, P. Santens, E. Mulleners, S. Engelborghs, P. P. De Deyn, J. Vandekerckhove, M. Rosseneu, and C. Labeur. 2000. Characterization and functional studies of lipoproteins, lipid transfer proteins, and lecithin:cholesterol acyltransferase in CSF of normal individuals and patients with Alzheimer's disease. J. Lipid Res. 41: 963-974. 
6. Fagan, A. M. and D. M. Holtzman. 2000. Astrocyte lipoproteins, effects of apoE on neuronal function, and role of apoE in amyloid-beta deposition in vivo. Microsc. Res. Tech. 50: 297-304.

7. Guyton, J. R., S. E. Miller, M. E. Martin, W. A. Khan, A. D. Roses, and W. J. Strittmatter. 1998. Novel large apolipoprotein E-containing lipoproteins of density $1.006-1.060 \mathrm{~g} / \mathrm{ml}$ in human cerebrospinal fluid. J. Neurochem. 70: 1235-1240.

8. Michikawa, M., Q. W. Fan, I. Isobe, and K. Yanagisawa. 2000. Apolipoprotein E exhibits isoform-specific promotion of lipid efflux from astrocytes and neurons in culture. J. Neurochem. 70: $1008-1016$.

9. Corder, E. H., A. M. Saunders, W. J. Strittmatter, D. E. Schmechel, P. C. Gaskell, G. W. Small, A. D. Roses, J. L. Haines, and M. A. Pericak-Vance. 1993. Gene dose of apolipoprotein E type 4 allele and the risk of Alzheimer's disease in late onset families. Science 261: 921-923.

10. Horsburgh, K., M. O. McCarron, F. White, and J. A. Nicoll. 2000. The role of apolipoprotein E in Alzheimer's disease, acute brain injury and cerebrovascular disease: evidence of common mechanisms and utility of animal models. Neurobiol. Aging 21: 245-255.

11. Weisgraber, K. H. and R. W. Mahley. 1996. Human apolipoprotein E: the Alzheimer's disease connection. FASEB J. 10: 1485-1494.

12. M. J. Ladu, C. Reardon, L. Van Eldik, A. M. Fagan, G. Bu, D. Holtzman, and G. S. Getz. 2000. Lipoproteins in the central nervous system. Ann. N. Y. Acad. Sci. 903: 167-175.

13. Spector, D. L. and H. C. Smith. Redistribution of U-snRNPs during mitosis. 1986. Exp. Cell Res. 163: 87-94. 
14. Kyrozis, A., C. Albuquerque, J. Gu, A. B.MacDermott. Ca(2+)-dependent inactivation of NMDA receptors: fast kinetics and high $\mathrm{Ca} 2+$ sensitivity in rat dorsal horn neurons. $1996 . J$. Physiol. 495 ( Pt 2): 449-463.

15. Hixson, J. E. and D. T. Vernier. 1990. Restriction isotyping of human apolipoprotein E by gene amplification and cleavage with Hha I. J. Lipid Res. 31: 545-548.

16. Chomczynski, P. and N. Sacchi. 1987. Single-step method of RNA isolation by acid guanidinium thiocyanate-phenol-chloroform extraction. Anal. Biochem. 162: 156-159.

17. Shachter, N. S., T. Ebara, R. Ramakrishnan, G. Steiner, J. L. Breslow, H. N. Ginsberg, and J. D. Smith. 1996. Combined hyperlipidemia in transgenic mice overexpressing human apolipoprotein CI. J. Clin. Invest. 98: 846-855.

18. Bisgaier, C. L., O. P. Sachdev, L. Megna, and R. M. Glickman. 1985. Distribution of apolipoprotein A-IV in human plasma. J. Lipid Res. 26: 11-25.

19. Chisholm, J. W., A. K. Gebre, and J. S. Parks. 1999. Characterization of C-terminal histidine-tagged human recombinant lecithin:cholesterol acyltransferase. J. Lipid Res. 40: 15121519.

20. Nichols, A. V., Krauss RM, Musliner TA (1986) Nondenaturing polyacrylamide gradient gel electophoresis. Methods Enzymol. 128: 417-431.

21. Forte. T. M. and R. W.Nordhausen. 1986. Electron microscopy of negatively stained lipoproteins. Methods Enzymol. 128: 442-457.

22. Hocquette, J. F., B. Graulet, and T. Olivecrona. 1998. Lipoprotein lipase activity and mRNA levels in bovine tissues. Comp. Biochem. Physiol. B. Biochem. Mol. Biol. 121: 201-212. 
23. Merkel, M., J. Heeren, W. Dudeck, F. Rinninger, H. Radner, J. L. Breslow, I. J. Goldberg, R. Zechner, and H. Greten. 2002. Inactive lipoprotein lipase (LPL) alone increases selective cholesterol ester uptake in vivo, whereas in the presence of active LPL it also increases triglyceride hydrolysis and whole particle lipoprotein uptake. J. Biol. Chem. 277: 7405-7411.

24. Albers, J. J., J. Lin, and G. P. Roberts. 1979. Effect of human plasma apolipoproteins on the activity of purified lecithin: cholesterol acyltransferase. Artery 5: 61-75.

25. Emmanuel, F., A. Steinmetz, M. Rosseneu, R. Brasseur, N. Gosselet, F. Attenot, S. Cuine, S. Seguret, M. Latta, and J. C. Fruchart. 1994. Identification of specific amphipathic alphahelical sequence of human apolipoprotein A-IV involved in lecithin:cholesterol acyltransferase activation. J. Biol. Chem. 269: 29883-29890.

26. Zorich, N, A. Jonas., and H. J. Pownall. 1985. Activation of lecithin cholesterol acyltransferase by human apolipoprotein $\mathrm{E}$ in discoidal complexes with lipids. J. Biol. Chem. 260: $8831-8837$.

27. Cohen, R. D., L. W. Castellani, J. H. Qiao, B. J. Van Lenten, A. J. Lusis, and K. Reue. 1997. Reduced aortic lesions and elevated high density lipoprotein levels in transgenic mice overexpressing mouse apolipoprotein A-IV. J. Clin. Invest. 99: 1906-1916.

28. Fisher, E. A. and H. N. Ginsberg. 2002. Complexity in the secretory pathway: the assembly and secretion of apolipoprotein B-containing lipoproteins. J. Biol. Chem. 277: 1737717380.

29. Petit-Turcotte, C., S. M. Stohl, U. Beffert, J. S. Cohn, N. Aumont, M. Tremblay, D. Dea, L. Yang, J. Poirier, and N. S. Shachter. 2001. Apolipoprotein C-I Expression in the Brain in Alzheimer's Disease. Neurobiol. Dis. 8: 953-963. 
30. Shachter, N. S. 2001. Apolipoproteins C-I and C-III as important modulators of lipoprotein metabolism. Curr. Opin. Lipidol. 12: 297-304.

31. Drigalenko, E, S. Poduslo, and R. Elston. 1998. Interaction of the apolipoprotein E and CI loci in predisposing to late-onset Alzheimer's disease. Neurology 51: 131-135.

32. Xu, Y., L. Berglund, R. Ramakrishnan, R. Mayeux, C. Ngai, S. Holleran, B. Tycko, T. Leff, N. S. Shachter. 1999. A common Hpa I RFLP of apolipoprotein C-I increases gene transcription and exhibits an ethnically distinct pattern of linkage disequilibrium with the alleles of apolipoprotein E. J. Lipid Res. 40: 50-58.

33. McCoy, M. G., G. S. Sun, D. Marchadier, C. Maugeais, J. M. Glick, and D. J. Rader. 2002. Characterization of the lipolytic activity of endothelial lipase. J. Lipid Res. 43: 921-929.

34. Borensztajn, J, T. J. Kotlar, and B. J. McNeill. 1980. Uptake of phospholipid-depleted chylomicrons by the perfused rat liver. Biochem. J. 192: 845-851.

35. Crawford, S. E. and J. Borensztajn. 1999. Plasma clearance and liver uptake of chylomicron remnants generated by hepatic lipase lipolysis: evidence for a lactoferrin-sensitive and apolipoprotein E-independent pathway. J. Lipid Res. 40: 797-805.

36. Ghazanfari, F. A. and R. R. Stewart. 2001. Characteristics of endothelial cells derived from the blood-brain barrier and of astrocytes in culture. Brain Res. 890: 49-65.

37. Ripodas, A, J. A. de Juan, M. Roldan-Pallares, R. J. Bernal, Moya, M. Chao, A. Lopez, A. Fernandez-Cruz, and R. Fernandez-Durango. 2001. Localisation of endothelin-1 mRNA expression and immunoreactivity in the retina and optic nerve from human and porcine eye. Evidence for endothelin-1 expression in astrocytes. Brain Res. 912: 137-143. 
38. DeMattos, R. B., R. P. Brendza, J. E. Heuser, M. Kierson, J. R. Cirrito, J. Fryer, P. M. Sullivan, A. M. Fagan, X. Han, and D. M. Holtzman. 2001. Purification and characterization of astrocyte-secreted apolipoprotein E and J-containing lipoproteins from wild-type and human apoE transgenic mice. Neurochem. Int. 39: 415-425.

39. Gregg, R. E., L. A. Zech, E. J. Schaefer, D. Stark, D. Wilson, and H. B. Brewer, Jr. Abnormal in vivo metabolism of apolipoprotein E4 in humans. 1986. J. Clin. Invest. 78: 815821.

40. Dong, L. M., C. Wilson, M. R. Wardell, T. Simmons, R. W. Mahley, K. H. Weisgraber, and D. A. Agard. 1994. Human apolipoprotein E. Role of arginine 61 in mediating the lipoprotein preferences of the E3 and E4 isoforms. J. Biol. Chem. 269: 22358-22365.

41. Bodovitz, S. and W. L. Klein. 1996. Cholesterol modulates alpha-secretase cleavage of amyloid precursor protein. J. Biol. Chem. 271: 4436-4440. 


\section{FIGURES}

Figure 1. Cholesterol in FPLC fractions of conditioned medium from human astrocytes cultured either in control serum-free medium or in medium supplemented with $16 \mu \mathrm{g} / \mathrm{ml}$ cholesterol.

A. Total cholesterol and free cholesterol (FC) in FPLC fractions of conditioned medium from cells cultured in control medium. Four peaks are evident. Cholesterol was predominantly esterified in all peaks, though significant free cholesterol was present in Peak 1.

B. Total cholesterol (TC) in FPLC fractions of medium from cells cultured in control (con) medium and in medium from cells cultured with supplemental cholesterol (ch). Cholesterol increased in Peak 1 and Peak 4 after cholesterol supplementation of cells.

C. Triglycerides (TG) in FPLC fractions of medium from cells cultured either in control serum-free medium or in medium supplemented with $16 \mu \mathrm{g} / \mathrm{ml}$ cholesterol. TG in lipoproteins was little altered after cholesterol supplementation.

Figure 2. A. Cholesterol and TG in FPLC fractions of serum-free medium conditioned by human astrocytes from a second donor (Donor 2). Cholesterol and TG are primarily detected in Peak 1 and Peak 4.

B. Cholesterol and TG in FPLC fractions of serum-free medium conditioned by human astrocytes from a third donor. Four peaks are seen that are similar in size. TG were present at about half the level of cholesterol in Peak 1, present equally to cholesterol in Peak 2 and in Peak 3 and present at about double the level of cholesterol in Peak 4. 
Figure 3. A. Immunoblot of human lecithin:cholesterol acyltransferase (LCAT) in astrocyte-conditioned medium from Donor 1 (Astrocytes) and in normal human plasma.

B. Immunoblot of apolipoprotein A-IV in astrocyte conditioned medium (Astrocyte medium) from Donor 1 and normal human plasma.

C. Immunoblot of human apoC-1 in pooled Peaks 3 and 4 and in Peak 1 FPLC fractions of cholesterol-supplemented $(\mathrm{CH})$ and control (Con) astrocyte-conditioned medium from Donor 1.

Figure 4. RNase Protection Assay of human apoC-I mRNA and 18S rRNA (control) in control human astrocytes from Donor 1 and in astrocytes cultured in medium supplemented with $16 \mu \mathrm{g} / \mathrm{ml}$ cholesterol $(\mathrm{CH})$. An RNA sample from a human apoC-I transgenic mouse was used as a positive control (Tg ApoC-I brain).

Figure 5. A. Immunoblot of Human ApoJ. Conditioned medium from human astrocytes from Donor 1 cultured either in control serum-free medium or in medium supplemented with $16 \mu \mathrm{g} / \mathrm{ml}$ cholesterol; $40 \mu \mathrm{g}$ of total protein (of each) was loaded.

B. Immunoblot of Human ApoE. Conditioned medium from astrocytes from Donor 1 cultured either in control serum-free medium or in medium supplemented with $16 \mu \mathrm{g} / \mathrm{ml}$ cholesterol; $70 \mu \mathrm{g}$ of total protein (of each) was loaded.

Figure 6. A. ApoE in FPLC lipoprotein fractions from Donor 2. VLDL: pooled lipoproteins from fractions 3, 4, 5 and 6. HDL: lipoproteins from fraction 31. HDL 2: pooled lipoproteins from fraction 35-38 (small HDL). ApoE3: apoE3 purified protein (control). 
B. ApoE, ApoJ and apoA-IV in pooled FPLC lipoprotein fractions from Donor 3. Peak 1 ("1") corresponds to VLDL, Peak 2 ("2") to LDL, Peak 3 (“3") to HDL, and Peak 4 (“4") to small HDL.

Figure 7. Non-denaturing gradient gel electrophoresis size and electron microscopic morphology of astrocyte VLDL (A) and HDL (B). VLDL on EM were heterogeneous in size, $40-180 \mathrm{~nm}$, diameter, consistent with non-denaturing gradient gel distribution where peak 1 is $40.3 \mathrm{~nm}$ and peak 2 is $38.3 \mathrm{~nm}$. Larger particles remained at the top of the gel. HDL noted in (B) consisted of three distinct peaks on gradient gels where 1, 2 and 3 correspond to 9.9, 9.3 and $8.5 \mathrm{~nm}$, respectively; the electron microscopic particles are also heterogeneous in size. Bar markers represent $100 \mathrm{~nm}$.

Figure 8. RT-PCR of Human Astrocyte RNA for secreted TG lipases. RT-PCR was performed for lipases as labeled. In each gel, the first lane represents a negative (water) control (-), the second human astrocyte RNA (HA), and the third a positive control RNA $(+)$.

Figure 9. A. Cholesterol in FPLC fractions of mouse astrocyte media. The mean of three separate single-donor preparations is shown with indicated standard deviations.

B. TG in the same FPLC fractions of mouse astrocyte media. C. Western Blot showing apolipoproteins E, J and A-IV in pooled lipoprotein peaks from mouse astrocyte media, mouse plasma as control.

Figure 10. Immunoblot for mouse LCAT in concentrated mouse astrocyte conditioned medium and mouse plasma. Three replicates of medium and two of plasma are shown. 


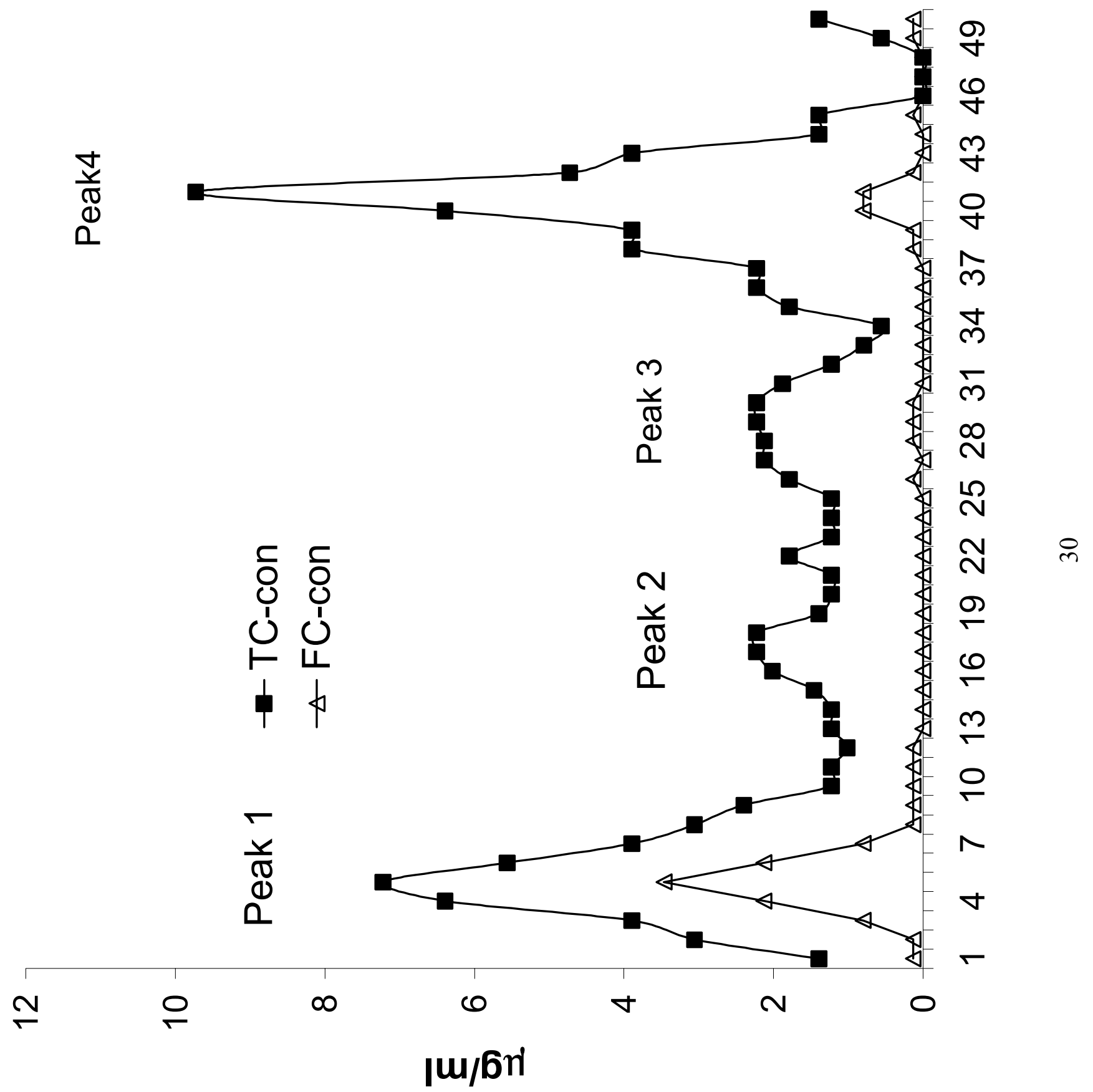

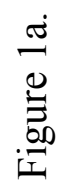




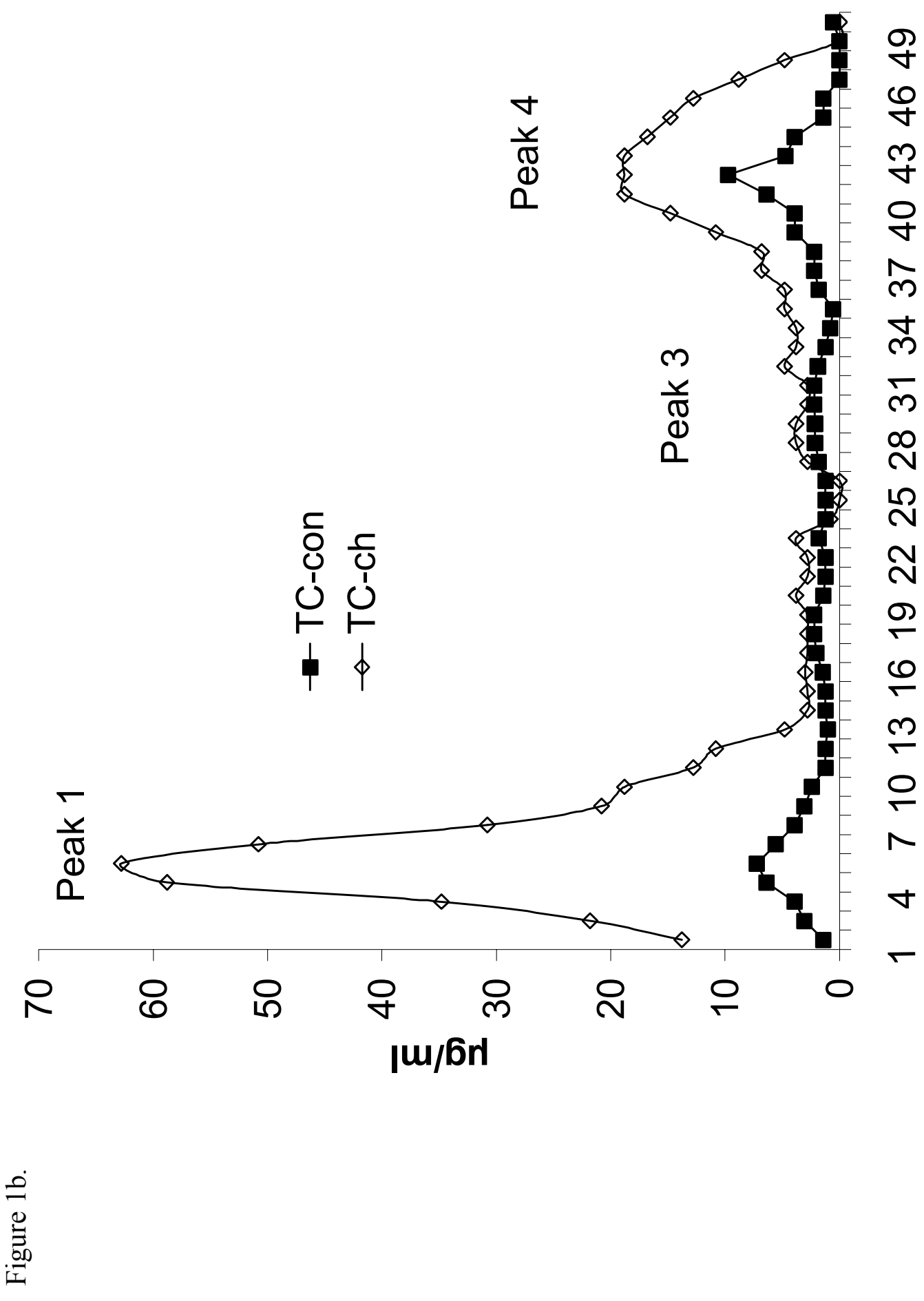




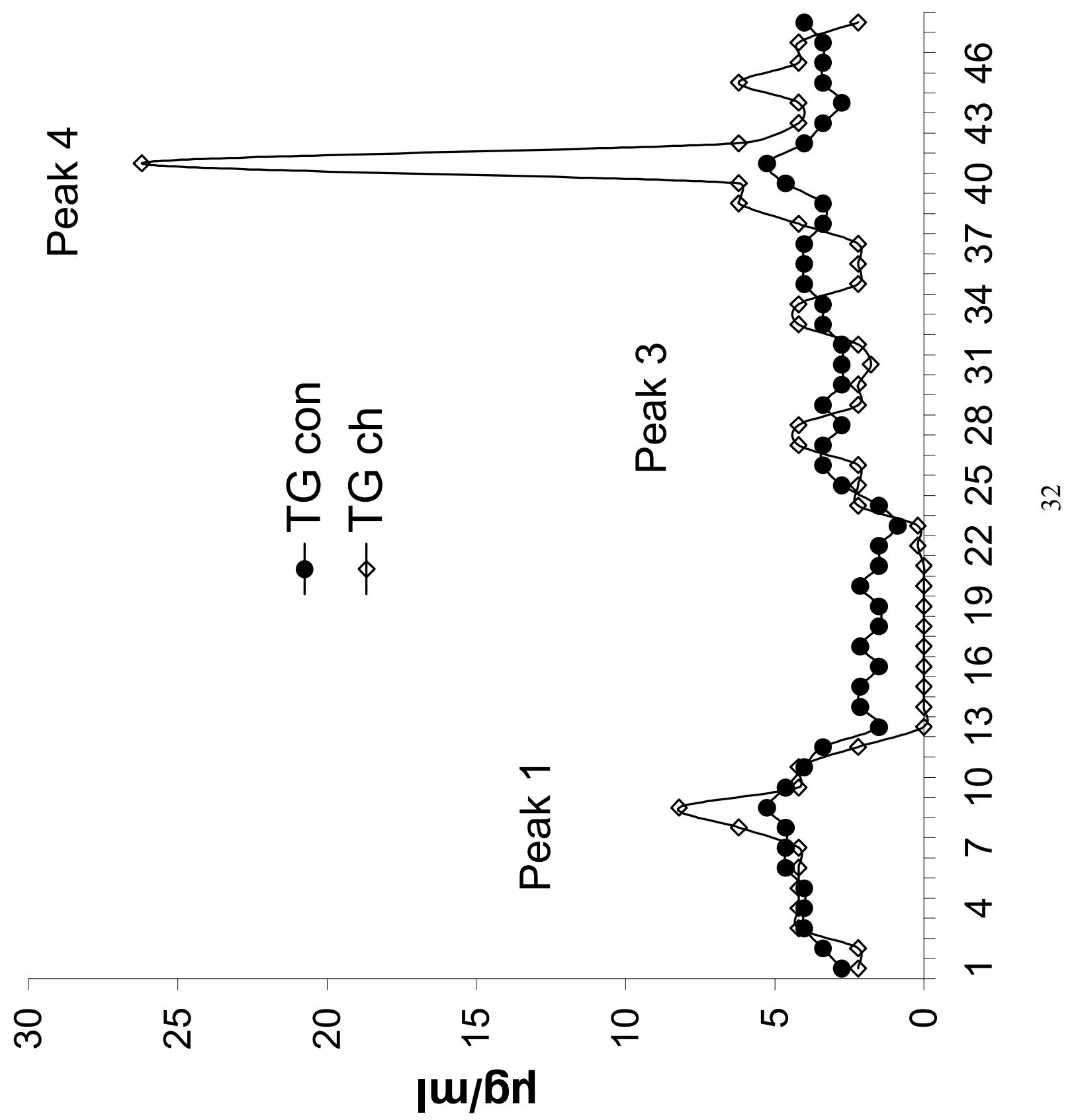

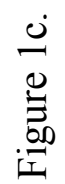




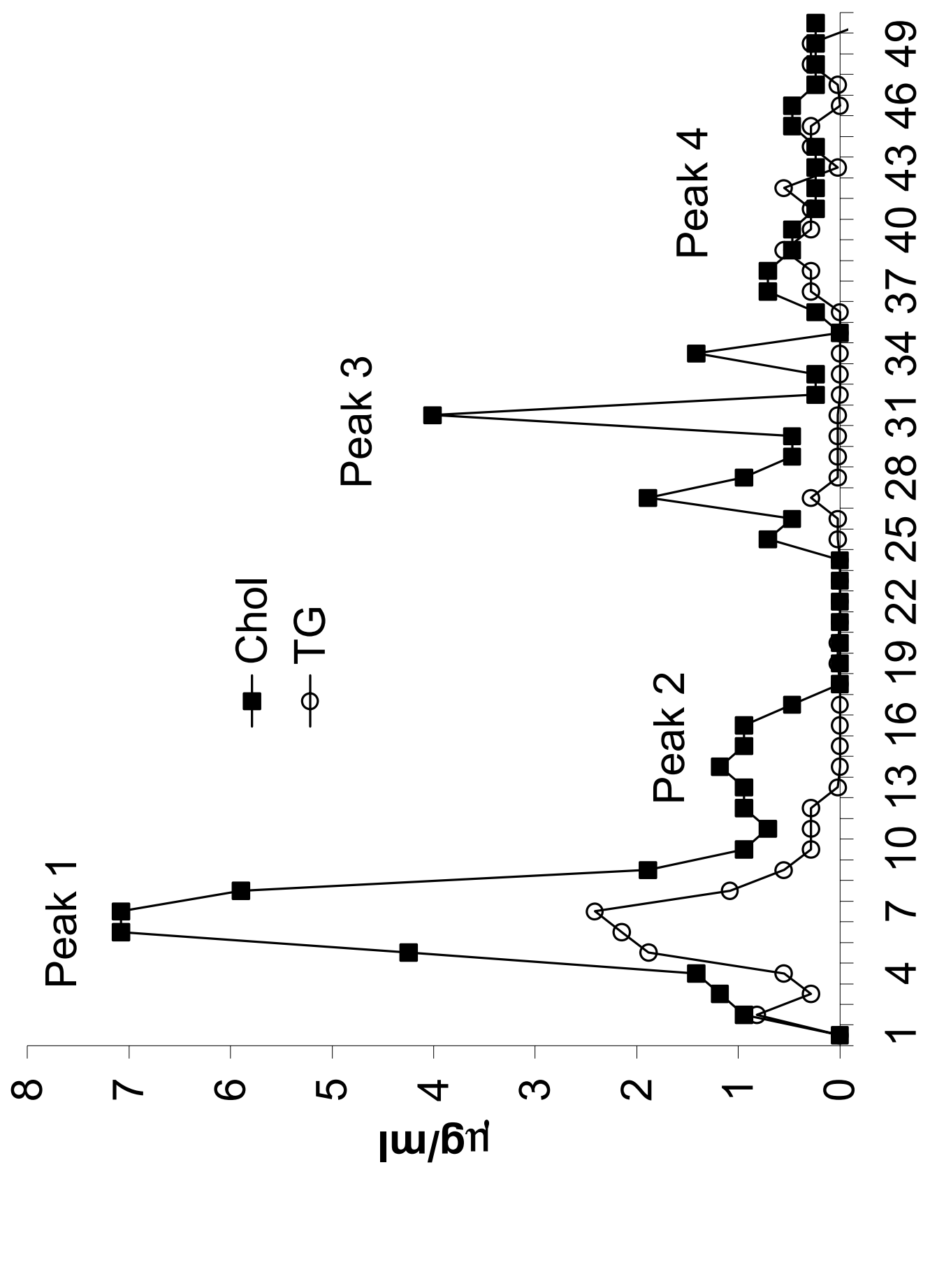




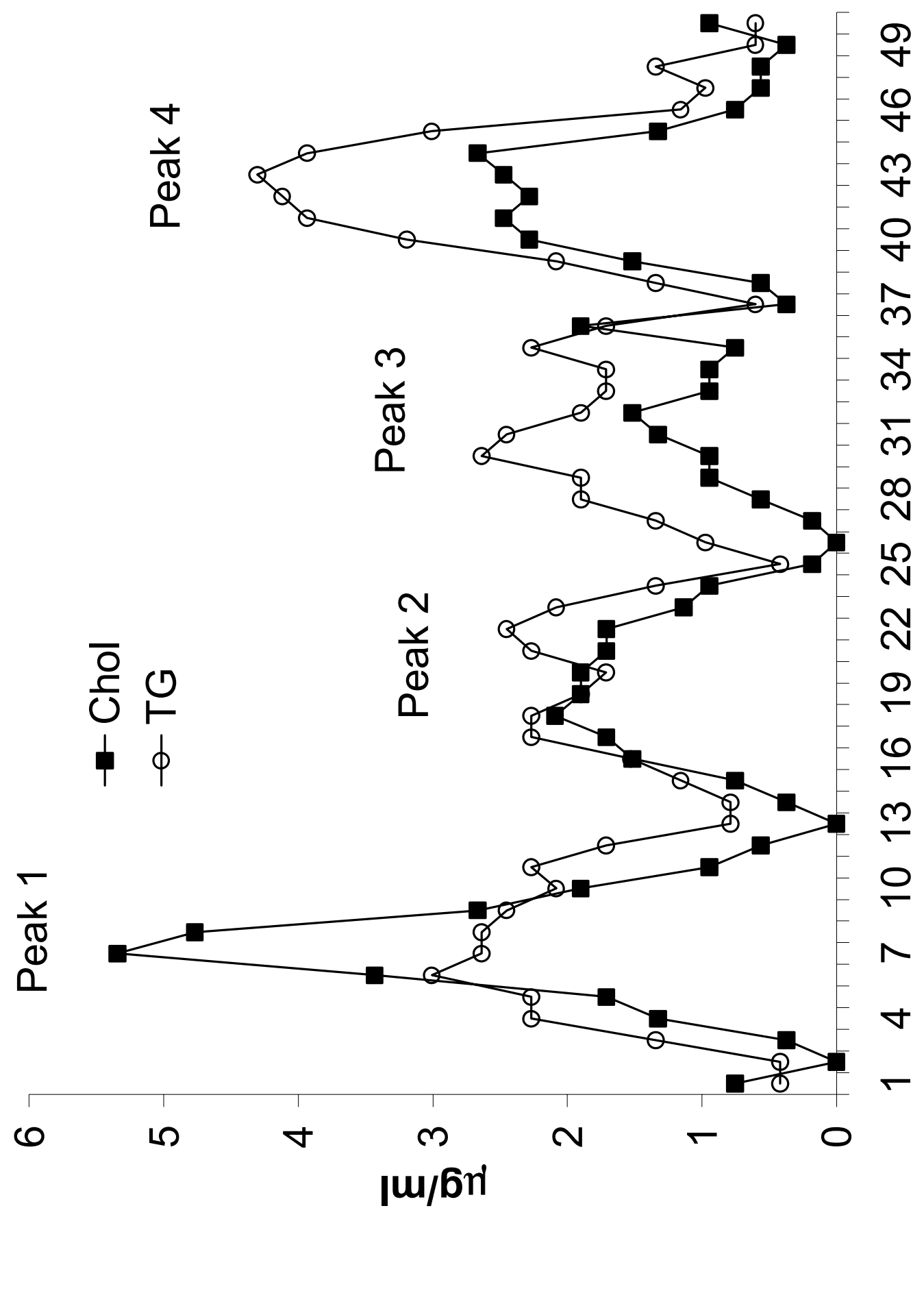



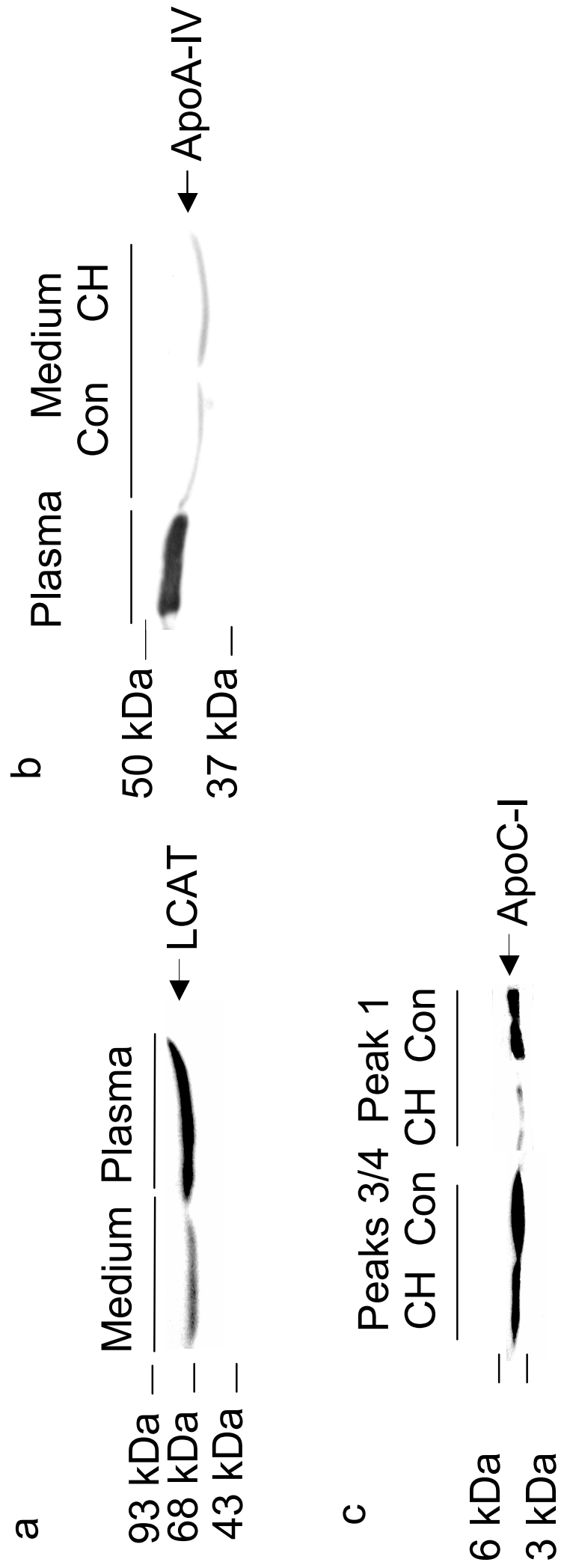


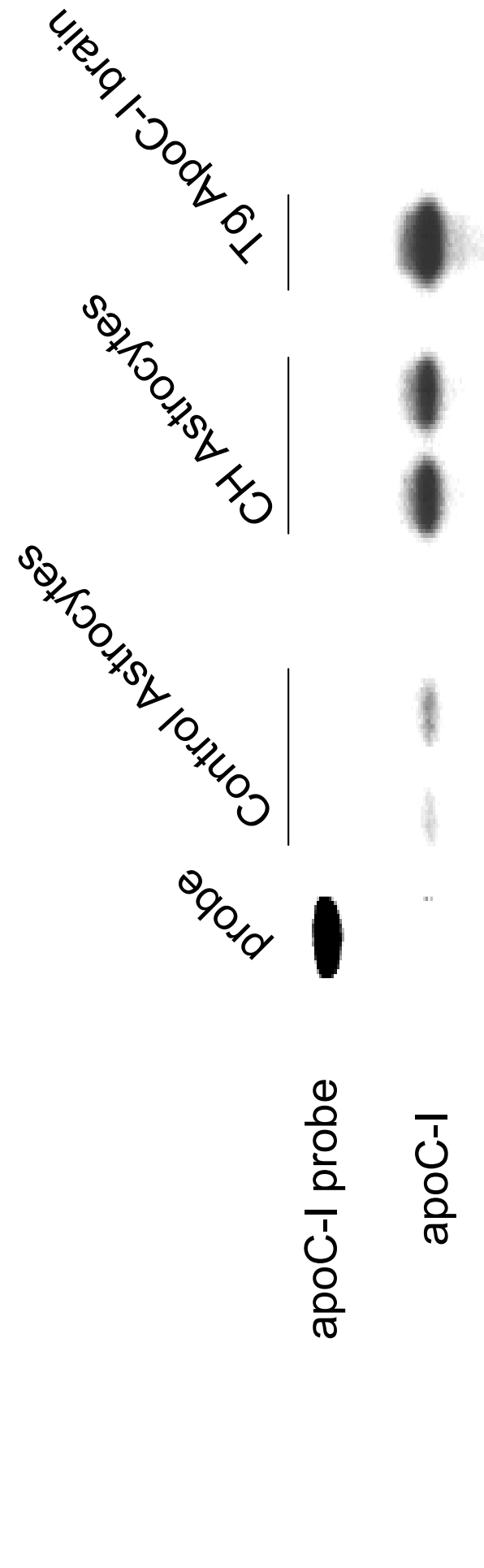

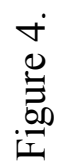




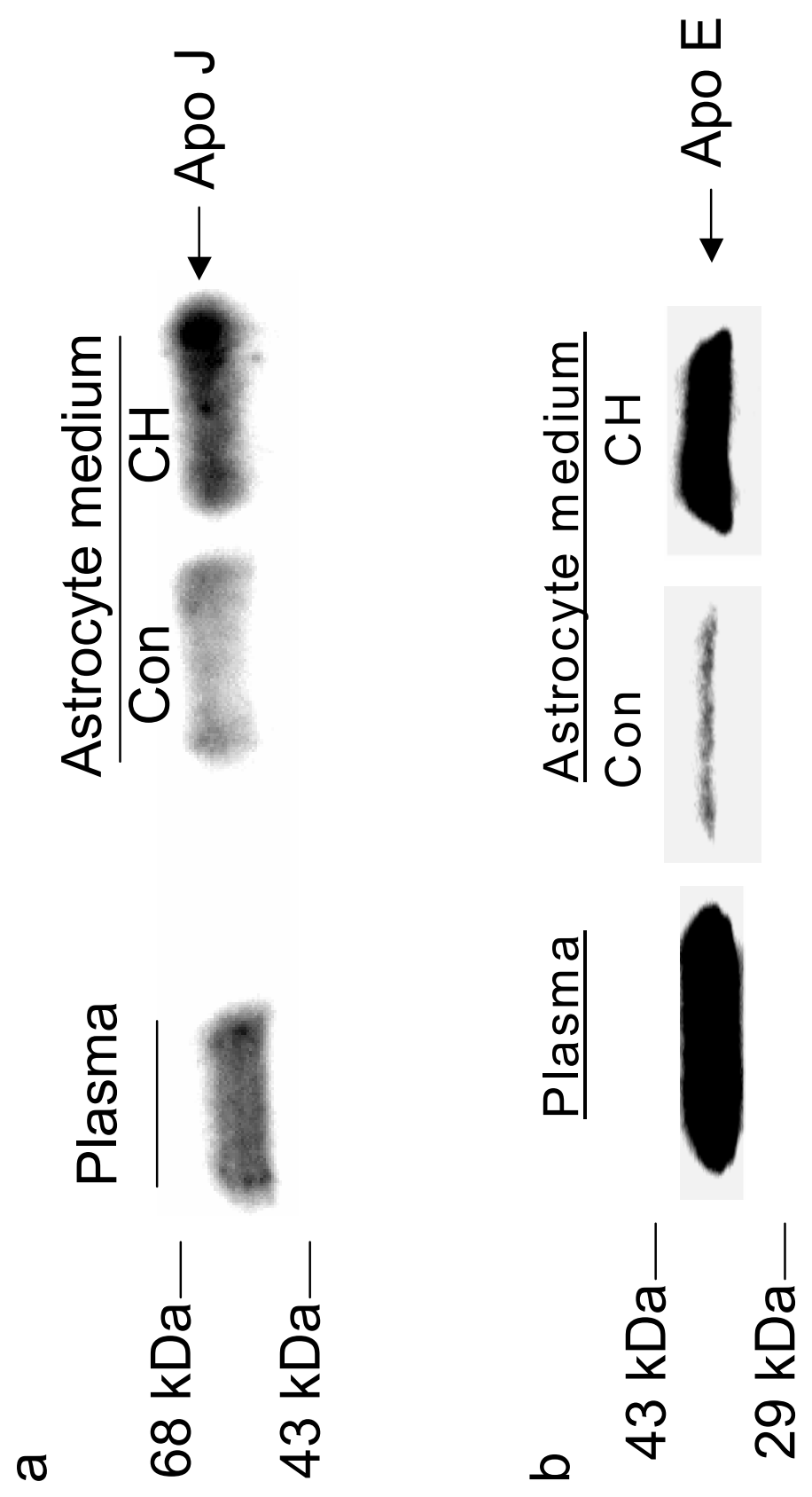

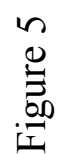



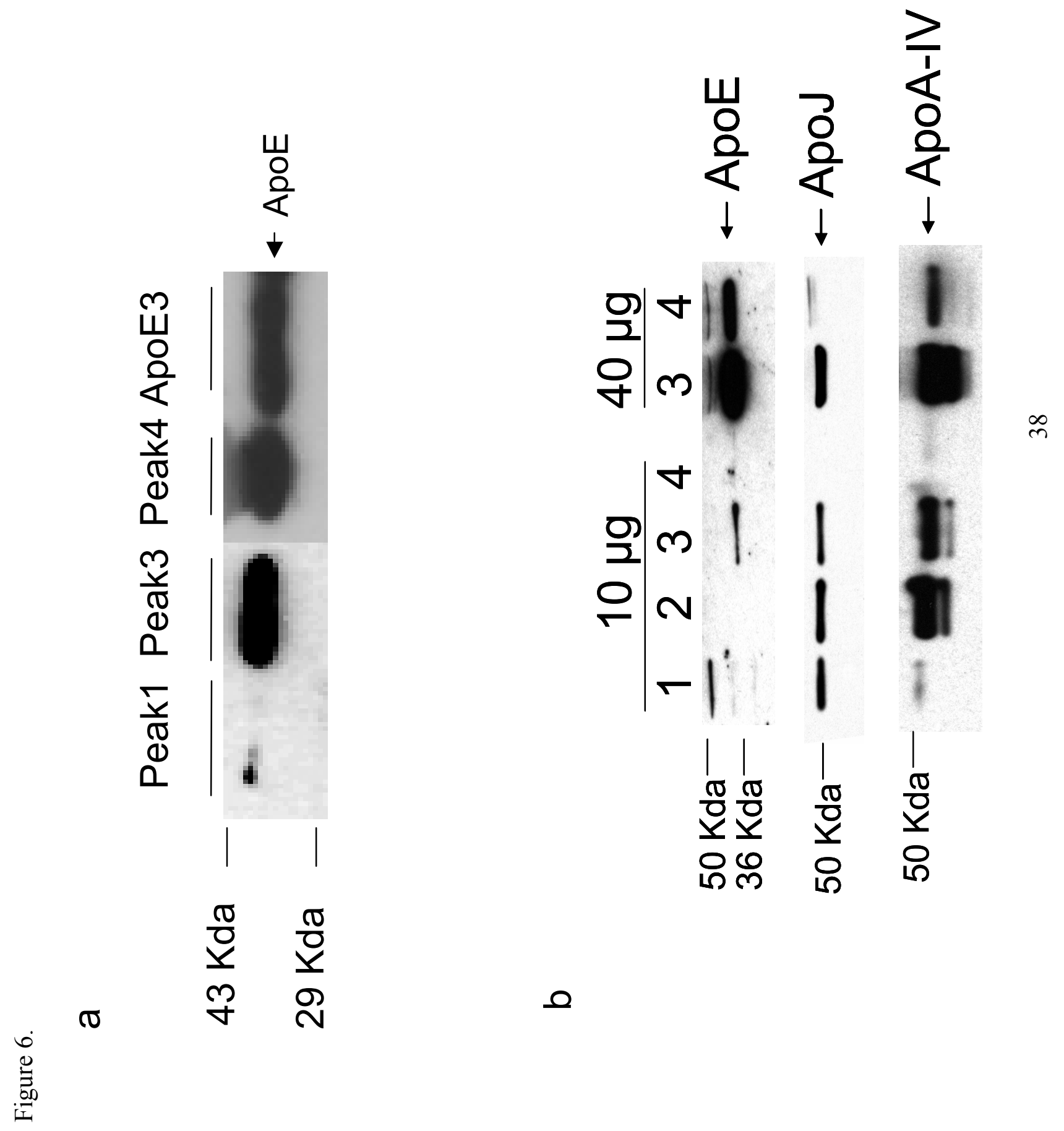

○ 

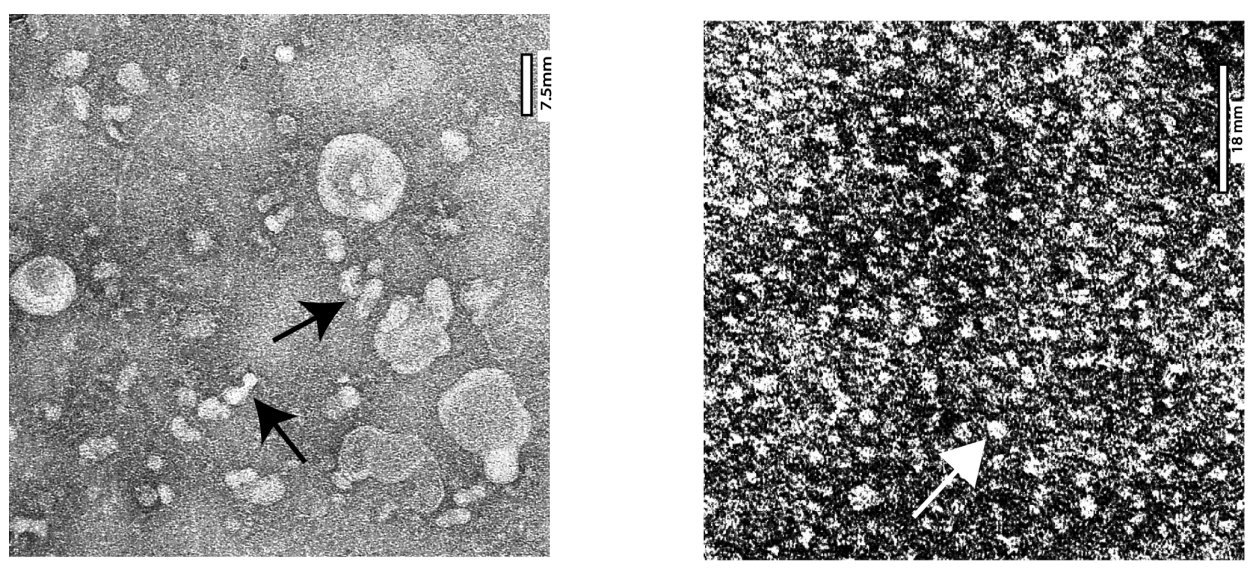

ले
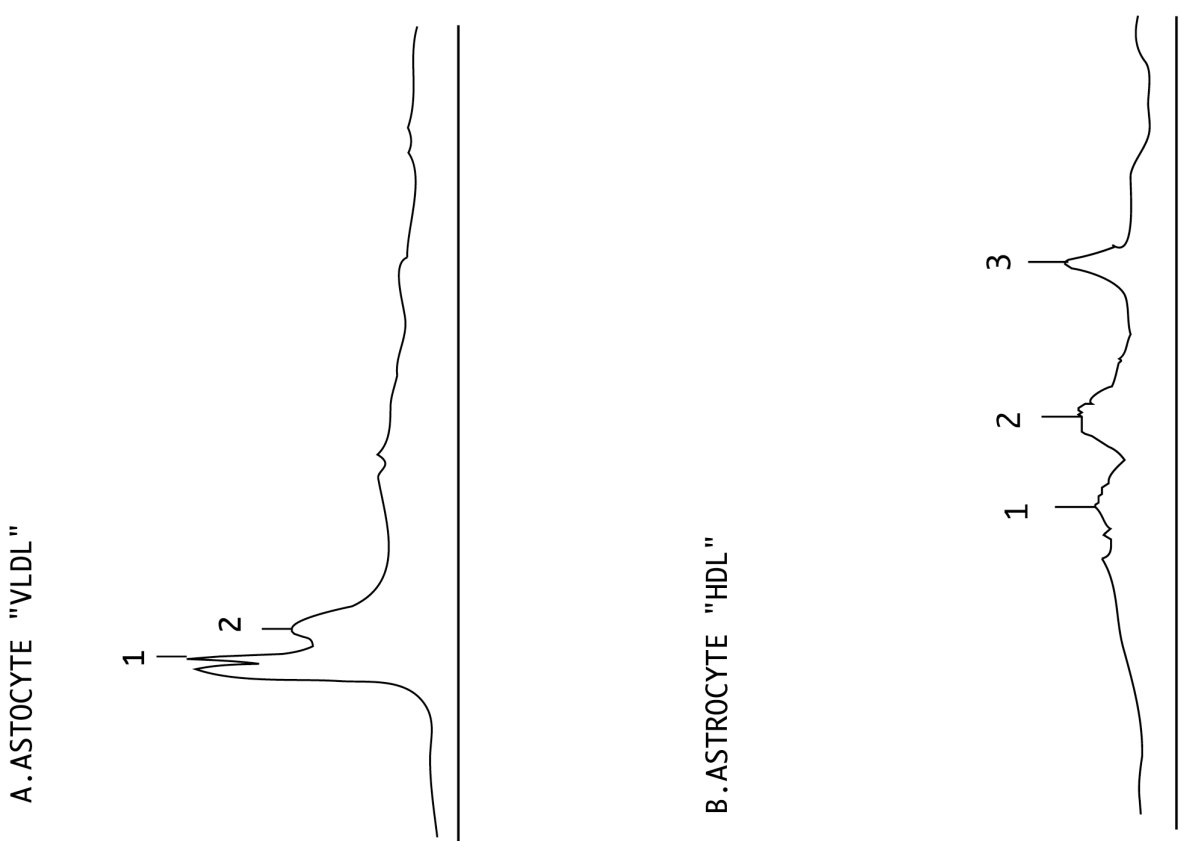

范 

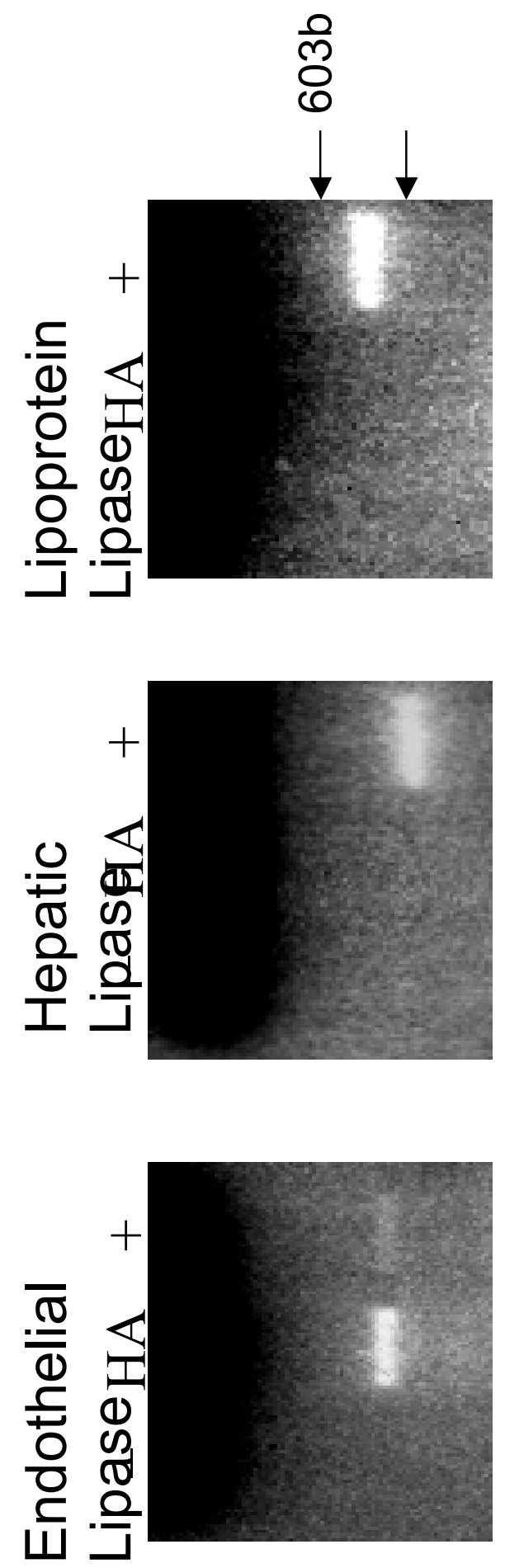


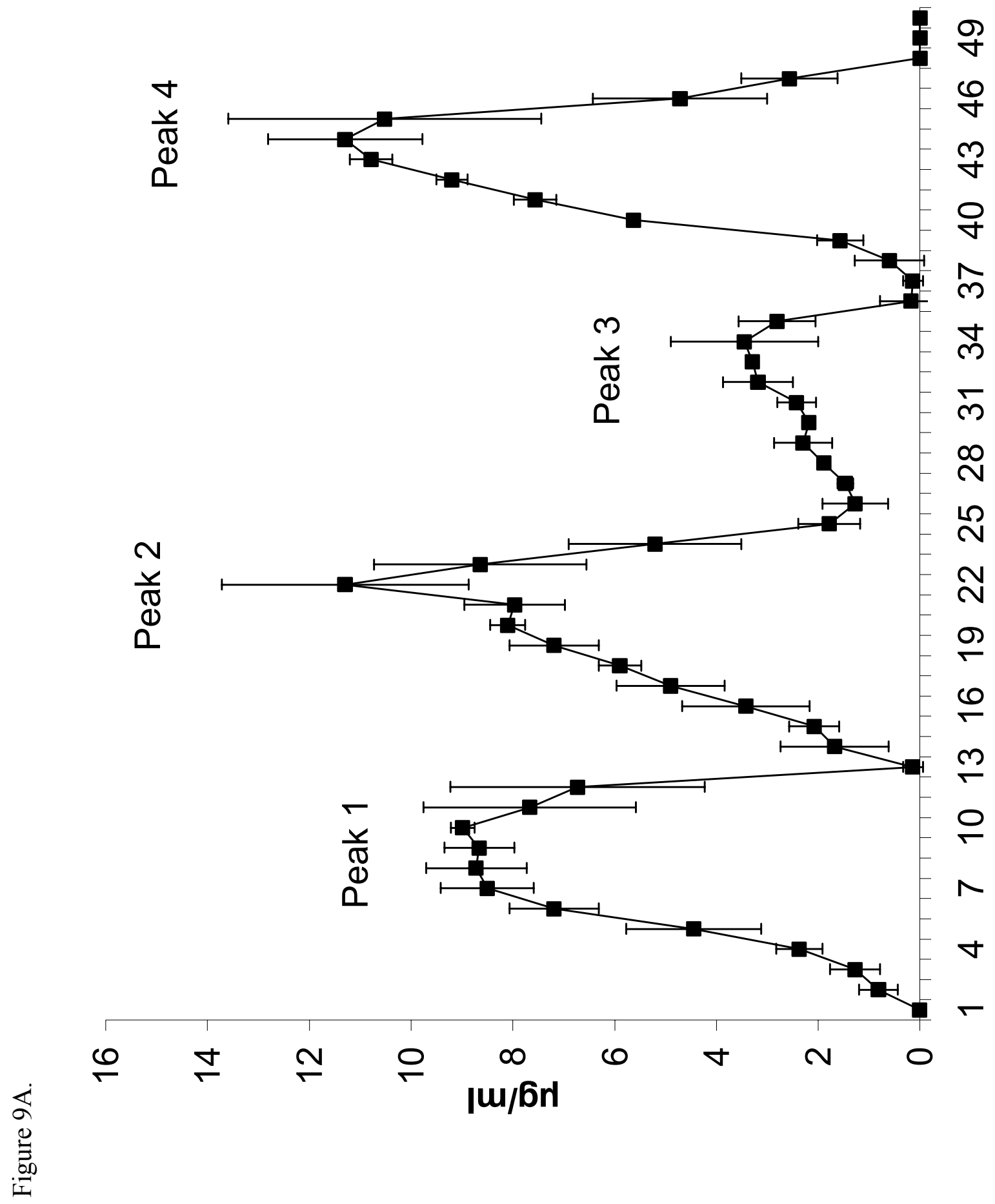




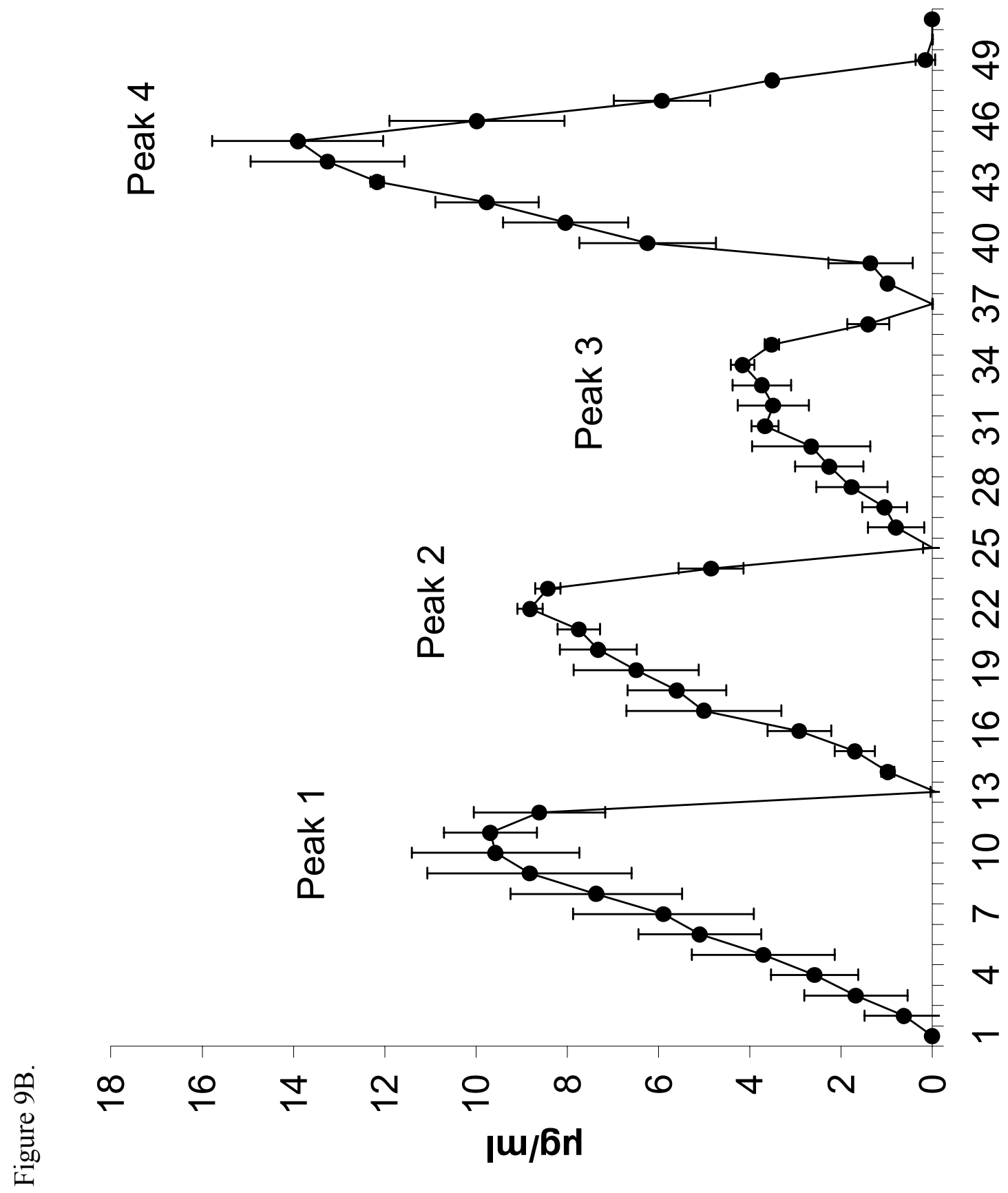




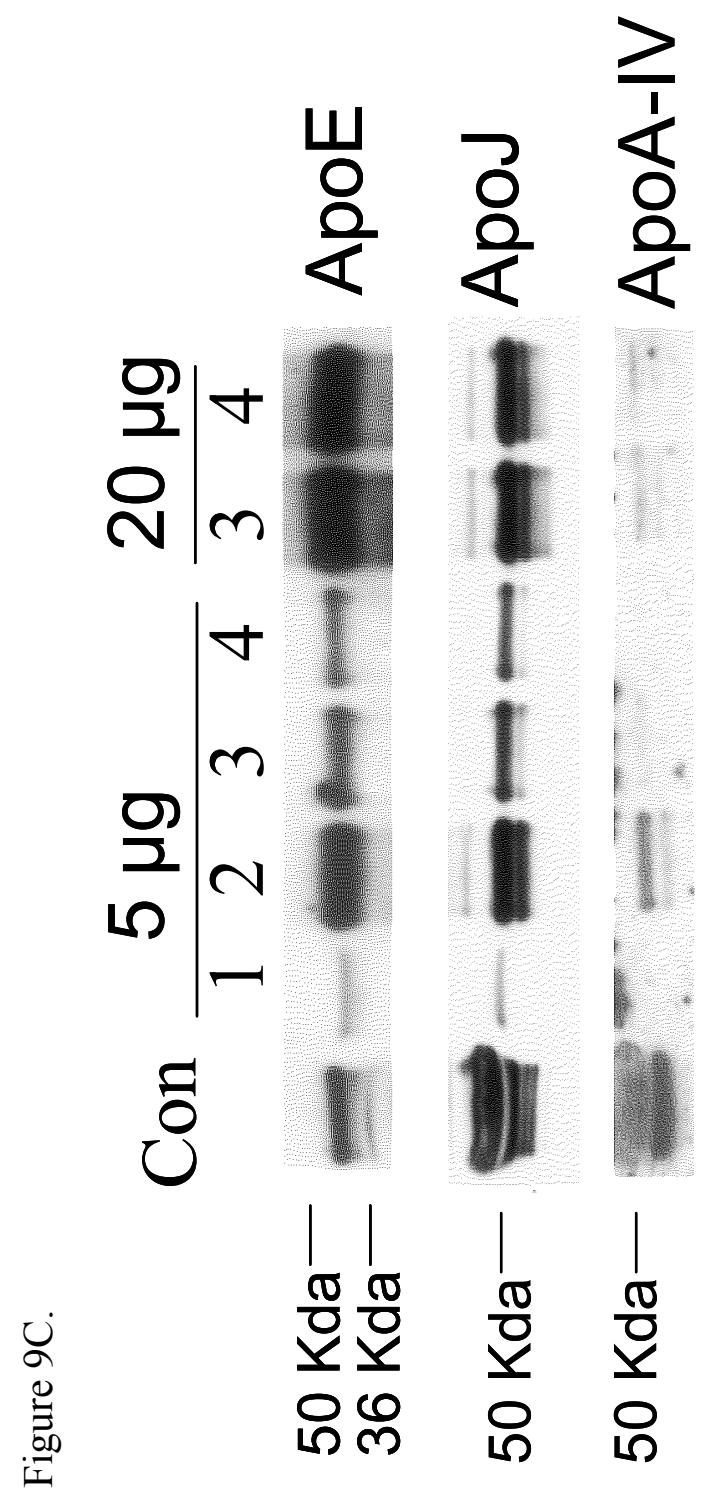




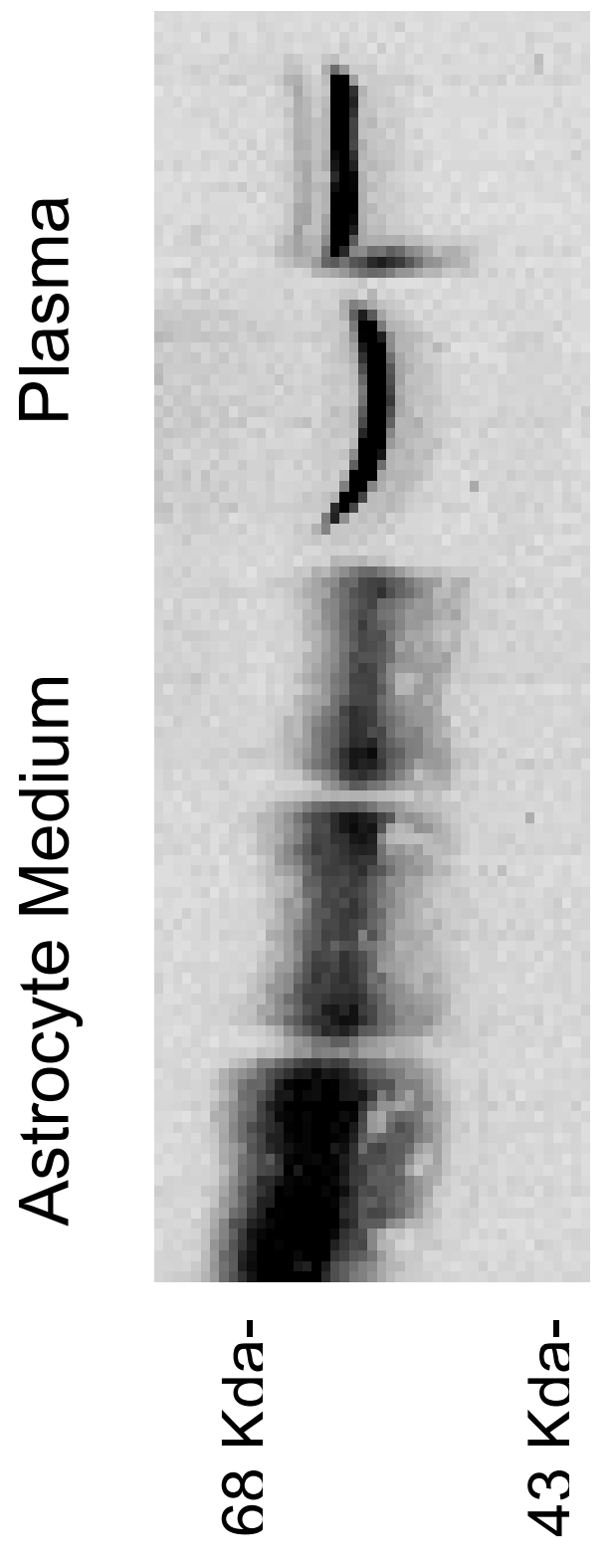

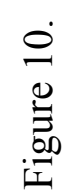

$\frac{1}{8}$ m 